\title{
The Mythical Mitochondrial Function in Lung Health and Disease
}

ISSN: 2578-0093

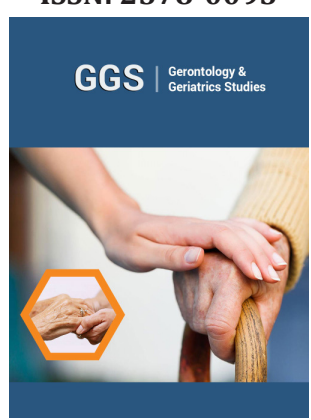

*Corresponding author: Arturo Solís Herrera, Human Photosynthesis Research Center. Aguascalientes 20000, México

Submission: 眥 November 07, 2020

Published: 眥 November 18, 2020

Volume 6 - Issue 3

How to cite this article: Arturo Solís Herrera. The Mythical Mitochondrial Function in Lung Health and Disease. Gerontol \& Geriatric stud. 6(3). GGS. 000638. 2020.

DOI: $10.31031 /$ GGS.2020.06.000638

Copyright@ Arturo Solís Herrera, This article is distributed under the terms of the Creative Commons Attribution 4.0 International License, which permits unrestricted use and redistribution provided that the original author and source are credited.

\section{Arturo Solís Herrera}

Human Photosynthesis Research Center. Aguascalientes 20000, México

\begin{abstract}
Mitochondria is described as various key functions such as cell cycle regulation, proliferation, apoptosis, and innate immune responses, in addition to power generation. The mitochondrion is relatively simple as consists of the outer membrane, inner membrane, proteins, lipids, and mitochondrial DNA that has substantial similarity to bacterial DNA. Mitochondrial morphology is in a dynamic state being modified continuously enabling the organelle to move, fuse, and fission depending on functional requirements of the cell, Although the changing states of mitochondria are described in detail, researchers are usually silent about the energy needed to maintain such dynamics. Mitochondria disorders are quite common in many diseases, such as neurodegenerative diseases. Compared to other organs such as heart, brain and liver, the lung has fewer mitochondria. Theoretically lungs rely on glycolysis more than oxidative phosphorylation for energy production. Furthermore, the role of mitochondria in normal lung homeostasis and importance of mitochondrial dysfunction/damage in the pathology of lung diseases remain poorly understood.
\end{abstract}

Keywords: Mitochondria; Energy; ATP; Homeostasis; Hydrogen; Oxygen; Water dissociation; COVID-19; Hyperoxia

\section{Background}

Mitochondria are key cellular organelles that not only supply cellular ATP but also integrate redox signaling, apoptotic balance, and biosynthetic pathways in the cell. However, mitochondria are not self-sufficient in relation to energy, so, for so many functions described the question is where the energy that mitochondria requires to perform so many key functions comes from? Supposedly, mitochondrial dysfunction leads to loss of cellular function, and in humans, mitochondrial dysfunction causes numerous pathologies including cancer, cardiovascular disease, neurological disorders, and respiratory diseases. Mitochondria are a major source of cellular reactive oxygen species (ROS), and accumulation of mitochondriaderived ROS have been linked to several human diseases. Thereby, as mitochondrial ROS is linked to mitochondrial dysfunction in various human pathologies it is particularly important the role of antioxidants.

The origin of mitochondria in eukaryotes is controversial, as mitochondria share several features with prokaryotes. The endosymbiotic theory proposes the generally accepted notion that mitochondria originated as an independent organism that took up residence in eukaryotic cells as endosymbionts [1]. Mitochondria regulate various metabolic functions and generate $>95 \%$ of the required metabolic energy that is driven by both nuclear and mitochondrial genomes; however, the mystery remains about where the energy that mitochondria needs to carry out its delicate functions where it comes from comes from?

Mitochondrial ROS production is tightly regulated and is generated by one electron reduction of molecular 02 to yield superoxide $\left(\mathrm{O}_{2} \cdot \mathrm{O}^{-}\right)$that is subsequently converted to hydrogen peroxide $\left(\mathrm{H}_{2} \mathrm{O}_{2}\right)$ by mitochondrial manganese-dependent superoxide dismutase $\left(\mathrm{SOD}_{2}\right) . \mathrm{O}_{2}$. generated within the inner mitochondrial membrane is not diffusible due to is polar; however, $\mathrm{H}_{2} \mathrm{O}_{2}$ produced by $\mathrm{SOD}_{2}$ easily negotiates membranes and enters cytoplasm where it enters several oxidative reactions. It gets decomposed to $\mathrm{H}_{2} \mathrm{O}$ and $\mathrm{O}_{2}$ by catalase or utilized by glutathione peroxidase or other peroxidases to oxidize their substrates. But the regulation of functions requires energy, the addition of an electron to molecular oxygen requires energy, the subsequent conversion to hydrogen peroxide also requires energy, and the action of superoxide dismutase also requires energy, and the literature is silent about it. 


\section{Introduction}

The role of mitochondria in normal lung homeostasis and its importance in the pathology of lung diseases remain poorly understood. The apparently well-recognized role of mitochondria in the generation of cellular energy is in fact poorly understood. The term ROS refers to a variety of reactive molecules derived from $\mathrm{O}_{2}$ such as $\mathrm{O}_{2}$., hydroxyl radical (OH.), or $\mathrm{H}_{2} \mathrm{O}_{2}$. Similarly, RNS refers to reactive species derived from $\mathrm{N}_{2}$ and $\mathrm{O}_{2}$ that include peroxynitrite $\left(\mathrm{ONOO}^{-}\right)$and nitric oxide (NO). Of the various reactive species, $\mathrm{OH}$ and $\mathrm{ONOO}^{-}$are the most reactive and cytotoxic, while $\mathrm{NO}$ and $\mathrm{H}_{2} \mathrm{O}_{2}$ are less reactive. Mammalian systems have multiple pathways that could be responsible of the generation of these reactive species. These include mitochondrial electron transport chain, NADPH oxidase (NOX 1-5), cytochrome P450 enzymes, cyclooxygenases, lipoxygenases, amino acid oxidases, and xanthine oxidase [2].

Most ROS are generated in mammalian cells by the mitochondrial respiratory chain [3] and NOX proteins [4]. Additionally, mitochondrial respiratory chain can produce NO [5], which on interaction with $\mathrm{O}_{2}^{-}$. forms $\mathrm{ONOO}^{-}$, a powerful oxidant. Both ROS and RSN can oxidize macromolecules such as proteins, nucleic acids, and lipids, and RSN also can modulate nitration or nitration of cellular thiols, proteins, and polyunsaturated fatty acids [6]. However, the production of reactive species, whether oxygen or nitrogen, do not form randomly, because if the biochemical order inside the cells is preserved, not formed or almost un forms. Numerous theories are handled but none include energy flow, they only focus on the flow of mass. $\mathrm{O}_{2}$-generation within the mammalian mitochondrial matrix is governed primarily by $\mathrm{O}_{2}$ concentration and levels of reduced electron donors. Oxygen does not come from the atmosphere, as it cannot spread through lung tissues, since oxygen is a polar as oil and water is highly polar, so they cannot and are not mixed. Which is the main reason why diffusion does not explain the exchange of gases in the lung, which was first reported from 1897, by Haldane [7]. And despite the articles that have been published about it, neither the researchers, nor the authors of the textbooks, nor the clinicians, have been concerned with finding the explanation, and have merely perpetuated the collective error. Thus, $\mathrm{O}_{2}^{-}$. Generated supposedly at different sites of mitochondrial respiratory chain may have different physiological and pathophysiological functions, which require further studies, which implies that despite the denoted efforts of the researchers, there are still many contradictions in this regard. Another example of incognitos about metabolism is that at least 4 isoforms of nitric oxide synthase are described in mammalian cells. But the enzymes in which the ace suffix is used means that it does not use ATP to perform its function, as opposed to the suffix tetase, for instance aminoacyl ARNt sintetase, meaning that the enzyme so-called does use energy coming from the hydrolysis of ATP, GTP, CTP, or TTP, to perform its function. However, in nitric oxide synthase, the energy required by this enzyme to perform its function cannot been defined.
Mitochondria-generated ROS and RNS can dysregulate mitochondrial activity and function by hampering modulation of mitochondrial fusion, fission, mtDNA damage, lipid peroxidation, and electrical gradient [8], which could be explained in several ways, but in any system, when the problem is power the fault is widespread and that is what we are looking at. Mitochondrial failure is widespread, so we must think about energy first. Superoxide anion $\left(\mathrm{O}_{2}\right)$ is dismutase into hydrogen peroxide $\left(\mathrm{H}_{2} \mathrm{O}_{2}\right)$ by the mitochondrial superoxide dismutase (mtSOD). Here two questions arise: where does the energy that mtSOD requires to carry out its action come from? and secondly, where do hydrogens come from? Literature is silent about it.

Mammalian cells have evolved several antioxidant defense mechanisms such as SOD, catalase, glutathione peroxidase, peroxiredoxins, and thioredoxins to detoxify or scavenge excess mitochondrial and non-mitochondrial ROS. These antioxidant mechanisms have been extensively studied, but these reports lack the same thing: where does the energy needed to make them work come from? It seems that the authors are not aware of the fundamental role of energy in any chemical process. It has been developed Mitochondria-targeted antioxidant therapies: based on the theoretical central role of mitochondria and mitochondrial ROS in human pathologies. Thereby, several natural antioxidants such as vitamin $\mathrm{C}$, vitamin $\mathrm{E}$, rottlerin, curcumin, ginsenoside $\mathrm{Rb} 1$, and epoetin delta have been investigated both in vitro and in vivo for their antioxidant efficacy, and as expected, most of these were not found to be effective in attenuating mitochondrial ROS production in response to an environmental stimulus [9].

Antioxidants such as alpha-tocopherol, ubiquinone, piperidine nitroxide, and CP have been conjugated to the lipophilic cation, triphenyl phosphonium (TPP), to generate mitochondria-targeted small molecule antioxidants Mito-E2, Mito-Q, Mito-CP, and MitoTEMPOL [10], However, experiments yield confusing and not infrequently contradictory results.

\section{Pulmonary Disorders and Mitochondrial ROS}

Theoretically, Mitochondrion is a key organelle that draws out the latent energy present in substrates such as glucose, fatty acids, and amino acids to generate utilizable energy in the form of ATP. But the supposed mechanisms through which mitochondria recovers the energy stored in the covalent bonds of glucose and other compounds remain a mystery or at least poorly understood. Also, the ATP, so far regarded as the universal energy exchange currency, it requires reconstitution at least three times per minute, which implies energy that is not spoken of in the respective articles. Moreover, you do not have a concise idea about how ATP releases energy, at most it is said to be through conformational modification. An estimated $70 \mathrm{~kg}$ man requires about $10 \mathrm{~kg}$ of ATP every 24 hours, which is not metabolically possible, even if confusing terms such as ATP amplification are used. 
The bioenergetic status in pulmonary disorders is disturbed what is associated with increased ROS generation, antioxidant depletion, and mitochondrial dysfunction leading to organ dysfunction. The fundamental processes of power generation through water dissociation are astonishingly accurate, and it is surprising that they have not changed since the beginning of time. But when the generation and distribution of energy that comes from the dissociation of water is altered by contaminated water, polluted air, pesticides, herbicides, fertilizers, plastic metals, solvents, industrial wastes, emotional stresses, alcohol, etc., then the function and shape of cells and tissues is disorganized resulting in the spread of molecules that are not normally present or in small amounts. On the one hand, biochemistry is becoming increasingly confuse trying to explain fruitless how energy comes from glucose, and on the other hand simply ignores fundamental issues.

Acute lung injury (due to sepsis or ventilator-induced lung injury) and subacute lung injury (due to ionizing radiation-induced lung injury) share profound increases in vascular permeability as a key element driving increased morbidity and mortality. There is evidence demonstrating a potential link between sepsis and mitochondrial dysfunction both in septic patients and animal models of sepsis. Ultrastructural abnormalities in mitochondrial morphology including irregular cristae were reported from liver and skeletal muscle biopsies obtained from septic patients who died in ICU [11]. The various suppositions that have been postulated to explain the alterations in the number, form, and function of mitochondria are entirely theoretical.

However, we note that functional and anatomical alterations that occur along with mitochondrial dysfunctions are multiple, suggesting a widespread failure, characteristic of energy. It is very consistent that when the power supply fails, failures are widespread. In the case of mitochondria, we have that it is not energetically autonomous. Mitochondria depends on an energy that has not been determined, as it theoretically produces energy for other cellular organelles through ATP, but it turns out that the functioning of the mitochondria or cell nucleus is not driven by ATP. The dissociation of the water molecule, which occurs inside the melanin granules, which are mainly located in perinuclear space, limited by the cell nucleus membrane and externally by the rough endoplasmic reticulum, it is a consistent explanation for the energy source not only of the mitochondria and the cell nucleus, but of all organelles and is even an energy capable of explaining all cellular functions.

The dissociation of water that occurs inside melanin [12] is described as follows:

$$
2 \mathrm{H}_{2} \mathrm{O} \text { (liquid) } \rightarrow 2 \mathrm{H}_{2} \text { (gas) }+\mathrm{O}_{2} \text { (gas) } \rightarrow 2 \mathrm{H}_{2} \mathrm{O} \text { (liquid) }+4 \mathrm{e}^{-}
$$

Unlike chlorophyll, the dissociation of water inside melanin is reversible, generating 4 high-energy electrons for every two water molecules that are reformed. When liquid water is transformed in its gaseous components, the truly valuable product is hydrogen since it is the energy hauler par excellence in the entire universe. To date it was wrongly believed that Oxygen was the valuable product because supposedly our body used it to combine it with oxygen in a kind of graduated combustion, also theoretical. But if glucose were a source of energy, diabetics would fly. The discovery that our body possesses the amazing ability to dissociate the molecule from water, breaks into a thousand pieces the sacrosanct role of glucose, mitochondria, and ATP as sources of energy. Glucose is only the universal precursor to any organic molecule in the human body, animals, and plants, but it is not able to provide the energy that its own metabolism requires. Therefore, we can now clearly discern that glucose does not have a dual role in biology as an energy source and as a source of building blocks simultaneously. On the other hand, the role of mitochondria is in the field of temperature regulation which is complemented by ATP, because when hydrolyzed to ADP, energy is absorbed, and when reduced to ATP, energy is released, while regulating phosphate levels, which are particularly toxic.

Therefore, when the generation and distribution of energy that comes from the dissociation of the water molecule is disturbed, then other cellular processes are also altered, and the extent of the alterations depends on the degree of damage that this fundamental system of dissociation of water has suffered, which has not changed since the beginning of time, and which is characterized by being astonishingly accurate. So, going back to the lung and mitochondria, we have that the amount of mitochondria present in lung tissues is significantly lower, because gas exchange is a significant component in strict temperature control that characterizes organisms made up of eukaryotic cells. That is: the inlet and outlet of atmospheric air tends to decrease the temperature of tissues, so the presence of mitochondria is not so necessary, in the understanding that their role is in temperature regulation, even their resemblance to heat sinks of electron circuits is substantive.

In both acute lung damage and bronchial asthma, tissue alterations are numerous and characteristically produced by external agents, which is consistent with the fact that the generation and distribution of energy from the dissociation of the water molecule is sensitive to numerous external agents. Mitochondrial alterations were expected to become present in these and other diseases, as energy failures induce widespread alterations, including mitochondria, as the cell uses energy in many ways, not only to carry out its functions but even to preserve form. Human mitochondrial DNA defects have been observed to accumulate with age and in age-related neurodegenerative diseases such as Alzheimer's and Parkinson's, which are induced by free radicals and ROS. But it is logical because we are exposed to the toxics of the environment from birth, which gradually permeate the tissues causing disturbances in the astonishingly accurate process of dissociation of water, leading to cells and tissues being disorganized.

Disorganized cellular functions, tissues, organs and systems tends to grow over time because contact with contaminated water, contaminated air, and food with pesticides, herbicides, fertilizers, 
metals, plastics, industrial waste, solvents, etc., is all day. Although no mitochondrial DNA changes are known to date, age-dependent mitochondrial defects may play a critical role in determining the susceptibility to asthma in the elderly. Genes also require energy to preserve form and function, whether they are in the cell nucleus or inside the mitochondria. There is no organic structure that escapes the constant need for energy. The alterations that cause disturbances in the generation and distribution of energy from the dissociation of the water molecule, are unpredictable since they depend on the age, weight, size, sex, and the nature of the environmental toxics that affected us, because in each person it is different and adding the complicated problem that the toxics, once inside the body, combine with each other forming new toxics.

Increased numbers of mitochondria and changes in mitochondrial ultrastructure and mitochondrial swelling have been shown in the bronchial epithelium of asthmatic mouse models and human asthmatics [13]. Such alterations can be explained by the failure in energy generation, and it would not be uncommon for mitochondria to increase in number in the diseased lungs, as frequent bacterial infections that are added tend to raise the temperature of tissue, which would induce an increase in the number of mitochondria to regulate it.

Mechanisms underlying increased mitochondrial ROS production are unclear, but this is explainable because researchers focus primarily on biochemical and structural alterations in tissues, setting aside the analysis of energy flow that is critical. Although no specific therapy is currently available to minimize mitochondrial dysfunction in experimental models and human asthma, mitochondria-targeted antioxidants may be, theoretically, a promising approach; however, they have yet to be tested as antiasthmatic drugs. Another interesting example is Pulmonary arterial hypertension $(\mathrm{PAH})$ is a lethal disease of the pulmonary vasculature characterized by pulmonary vasoconstriction, right ventricular hypertrophy, and right ventricular failure [14]. The hallmark of $\mathrm{PAH}$ is excessive proliferation of pulmonary artery smooth muscle cells that thicken the lumen and increase resistance in pulmonary arteries [15]. Etiology is therefore not understood as a complex disease, with a significant vascular component, genetic and environmental factors; hypoxia, viral infections, etc., but in the end the findings are compatible with a widespread failure, which should make us think about energy. Although mitochondrial dysfunction and mitochondrial ROS and RNS are implicated in PAH, potential antioxidant strategies specifically targeting mitochondrial ROS as therapy in PAH have been investigated only in animal models, and they need to be extended to humans. But if animal models were not energy-based, and specifically on the dissociation of the water molecule, then their results have been poor, so they have not spread to human patients.

Pulmonary fibrosis is a lung disease characterized by irreversible destruction of lung architecture, abnormal wound healing, and deposition of extracellular matrix proteins leading to organ dysfunction, disruption of gas exchange, and death from respiratory failure. Lung fibrosis could be idiopathic [16] or arise from exposure to environmental toxins such as fibers, asbestos, metals, pesticides, chemotherapeutic drugs, viruses, multiwalled carbon nanotubes, and radiotherapy [17]. Interestingly they are the same factors that affect the astonishingly precise dissociation of the water that occurs inside melanin, and the damage is widespread, which supports that the underlying problem is the generation and distribution of energy from melanin.

In the case of Bronchopulmonary dysplasia (BPD) is one of the devastating complications of premature newborns with no proven therapy. The development of BPD is associated with several risk factors including hyperoxia, ventilator-induced lung injury, and pre and postnatal infections [18]. An arrest of alveolar development, secondary to mitochondrial bioenergetics failure and reduced ATP production, is one of the hallmarks of BPD [19]. Alveolar development in the fetus requires an adequate, accurate energy supply for which melanin meets the requirements. Damage is also widespread, so failure in power generation and distribution is the most likely mechanism. Supplemental oxygen therapy (ventilator) resulting in oxygen toxicity, mediated, theoretically, by production and/or accumulation of ROS, has been implicated in the development of BPD in premature infants and experimental models of hyperoxia-induced injury [20]. Tissue damage is diffuse, so we need to think about energy first, and diffuse damage is usually difficult to explain and worst if we do not take energy flow into account and only study isolated structural alterations.

There is no clear explanation for the varying observations on the involvement of mitochondrial vs. NOX proteins in hyperoxiainduced ROS generation; however, use of different cell types, variation due to the use of mouse, rat, and humans, or differential levels of antioxidants in various cell types may all account for the discrepancies; however, the best antioxidant is molecular hydrogen. Although developmental differences in oxidative stress responses were observed in the murine lung, greater lethality of adult animals exposed to hyperoxia may be due to inflammation rather than to differences in AOEs [21]. Oxygen is a poisonous gas, and at high concentrations it is lethal. And intubating COVID-19 patients understand it, with 9 out of 10 dying. It is worrying that the medical body that cares for pandemic patients is not aware of the dangers posed by forced oxygen administration and the worst thing is that it is at high doses.

\section{Conclusion}

Mitochondria, often referred to "the powerhouses of the cell," play an important role in cellular redox status, signaling, innate immunity, aging, and homeostasis. However, we must bear in mind that mitochondria are not an energy-independent organelle, which means that it requires energy to carry out its delicate and exact functions, as well as to preserve form. On the other hand, we must dismiss the idea that glucose is an energy source. The idea that mitochondria by mysterious mechanisms can recover the energy stored in the covalent bonds of carbohydrates and other molecules and which eventually produce ATP, is one that is theoretical at 
95\%. Under stress or pathological situations, mitochondrial damage and dysfunction occurs, which can be induced by excess of mitochondrial ROS production and/or altered mitochondrial dynamics resulting in altered signaling pathways, modulation of transcriptional factors, immunity, and metabolic adaptation. but the main problem is in the dissociation of the water molecule and since it is the energy source of all cellular functions, the damage, as expected, is diffuse, is widespread. Increased mitochondria-derived ROS and mitochondrial dysfunction have been implicated in the pathogenesis of a variety of disorders including diabetes, cancer, and lung diseases such as ALI, asthma, pulmonary hypertension, lung cancer, lung fibrosis, and bronchopulmonary dysplasia. But in the end, they are different manifestations of the same problem which is the disturbance of the generation and distribution of energy that begins with the dissociation of the water molecule. Detoxification of mitochondrial ROS with antioxidants or antioxidant enzymes may be beneficial in ameliorating mitochondrial dysfunction, however, the results so far have been less than mediocre, as the mere restoration of adequate water dissociation levels is likely to dramatically improve the clinical picture.

\section{Acknowledgement}

This work was supported by Human Photosynthesis ${ }^{\oplus}$ Research Centre. Aguascalientes 20000, México.

\section{References}

1. Verschoor ML, Ungard R, Harbottle A, Jakupciak JP, Parr RL, et al. (2013) Mitochondria and cancer: past, present, and future. BioMed Res Int, Article ID 612369.

2. Turrens JF (2003) Mitochondrial formation of reactive oxygen species. J Physiol 552(Pt 2): 335-344.

3. Poyton RO, Ball KA, Castello PR (2009) Mitochondrial generation of free radicals and hypoxic signaling. Trends Endocrinol Metab 20(7): 332340.

4. Pendyala S, Natarajan V (2010) Redox regulation of Nox proteins. Respir Physiol Neurobiol 174(3): 265-271.

5. Castello PR, David P, Clure T, Crook Z, Poyton RO (2006) Mitochondrial cytochrome oxidase produces nitric oxide under hypoxic conditions: implications for oxygen sensing and hypoxic signaling in eukaryotes. Cell Metab 3(4): 277-287.

6. Baker PR, Schopfer FJ, Donnell VB, Freeman BA (2009) Convergence of nitric oxide and lipid signaling anti-infl ammatory nitro-fatty acids. Free Radic Biol Med 46(8): 989-1003.
7. John H, Lorrain SJ (1897) The absorption of oxygen by the lungs. Pathological Laboratory, Queens College, Belfast, UK.

8. Bolisetty S, Jaimes EA (2013) Mitochondria, and reactive oxygen species: physiology and pathophysiology. Int J Mol Sci 14(3): 6306-6344.

9. Alfadda AA, Sallam RM (2012) Reactive oxygen species in health and disease. J Biomed Biotechnol 2012: 936486.

10. Kelso GF, Porteous CM, Coulter CV, Hughes G, Porteous WK, et al. (2001) Selective targeting of a redox-active ubiquinone to mitochondria within cells: antioxidant and anti-apoptotic properties. J Biol Chem 276(7): 4588-4596.

11. Vanhorebeek I, Vos R, Mesotten D, Wouters PJ, Peeters CDW, et al. (2005) Protection of hepatocyte mitochondrial ultrastructure and function by strict blood glucose control with insulin in critically ill patients. Lancet 365(9453): 53-59.

12. Herrer AS, Esparza MCA, Ashraf G, Zamyatnin AA, Gjumrakch A (2015) Beyond mitochondria, what would be the energy source of the cell? Cent Nerv Syst Agents Med Chem 15(1): 32-41.

13. Mabalirajan U, Dinda AK, Kumar S, Roshan R, Gupta P, et al. (2008) Mitochondrial structural changes and dysfunction are associated with experimental allergic asthma. J Immunol 181(5): 3540-3548.

14. Montezano AC, Touyz RM (2012) Molecular mechanisms of hypertensionreactive oxygen species and antioxidants: a basic science update for the clinician. Can J Cardiol 28(3): 288-295.

15. Michelakis ED, Wilkins MR, Rabinovitch M (2008) Emerging concepts and translational priorities in pulmonary arterial hypertension. Circulation 118(14): 1486-1495.

16. Selman M, King TE, Pardo A (2001) Idiopathic pulmonary fibrosis: prevailing and evolving hypotheses about its pathogenesis and implications for therapy. Ann Intern Med 134(2): 136-151.

17. He X, Young SH, Berry DS, Chisholm WP, Fernback JE, et al. (2011) Multiwalled carbon nanotubes induce a fibrogenic response by stimulating reactive oxygen species production, activating NF-kB signaling and promoting fi broblast-to-myofi broblast transformation. Chem Res Toxicol 24(12): 2237-2248.

18. Baraldi E, Filippone M (2007) Chronic lung disease after premature birth. N Engl J Med 357: 1946-1955.

19. Ratner V, Starkov A, Matsiukevich D, Polin RA, Ten VS (2009) Mitochondrial dysfunction contributes to alveolar development arrest in hyperoxia-exposed mice. Am J Respir Cell Mol Biol 40(5): 511-518.

20. Northway WH, Rosan RC (1968) Radiographic features of pulmonary oxygen toxicity in the newborn: bronchopulmonary dysplasia. Radiology 91(1): 49-58.

21. Berkelhamer SK, Kim GA, Radder JE, Wedgwood S, Czech L, et al. (2013) Developmental differences in hyperoxia-induced oxidative stress and cellular responses in the murine lung. Free Radic Biol Med 61: 51-60. 